radiogram.

2) The diagnosis either between $L_{4}$ and $L_{5}$ or between $L_{5}$ and $S_{1}$ was able to make preoperatively with $100 \%$ of accuracy by the same methods.

3) However, among cases in the latter group the correct level diagnosis out of those two space was made in $86.5 \%$ of cases.

\begin{tabular}{c|c|c}
\hline number of case & preoperative diagnosis & postoperative diagaosis \\
\hline 10 & $\mathrm{~L}_{4}-\mathrm{s}$ & $\mathbf{L}_{4}-5, \mathrm{~L}_{5}-\mathbf{S}_{1}$ \\
6 & $\mathrm{~L}_{4}-5$ & $\mathrm{~L}_{5}-\mathrm{S}_{1}$ \\
6 & $\mathrm{~L}_{4}-\mathbf{S}_{1}$ & $\mathrm{~L}_{4}-5$ \\
11 & $\mathrm{~L}_{5}-\mathbf{S}_{1}$ & $\mathbf{L}_{4}-5, \mathbf{L}_{5}-\mathbf{S}_{1}$ \\
\hline
\end{tabular}

4) The important signs for neurological level diagnosis are atrophy and hypotony of muscles $(86.5 \%)$, tension signs $(87.2 \%)$, decrease of ankle jerk $(68.0 \%)$, tenderness of the cranial gluteal nerve $(86.7 \%)$, hypoaesthesia in the dorsum of the foot $(78.6 \%)$ and decrease of the powers of extension and flexion of the big toe (70\%).

However, there are no definite signs which differenciate between single disc protrusion either in $\mathrm{L}_{4}-5$ or $\mathrm{L}_{5} \mathrm{~S}_{1}$ and double protrusion both in $\mathrm{L}_{4-5}$ and $L_{5}-S_{1}$.

5) Narrowness of the intervertebral space in lateral view of radiogram also presents a positive finding for level diagnosis no less than neurological signs and was reliable in $100 \%$ between $\mathrm{L}_{3}$ and $\mathrm{L}_{4}, 96.2 \%$ between $\mathrm{L}_{4}$ and $\mathrm{L}_{5}, 65.6 \%$ between $\mathrm{L}_{5}$ and $\mathrm{S}_{1}, 84.6 \%$ both in $\mathrm{L}_{4}-5$ and $\mathrm{L}_{5}-\mathrm{S}_{1}$.

6) When myelography was not done, at the time of surgery both level of $\mathrm{L}_{4}-5$ and $\mathrm{L}_{5}-\mathrm{S}_{1}$ should be exposed by the romoval of the interarcuate ligament to avoid the wrong diagnosis $(13.5 \%)$, and with due regard to double protrusion $(26 \%)$.

\title{
116. Discogram of the Cervical Disc Lesion
}

\section{The First Report: Discogram of the Cervical Spondylosis}

\author{
Kiyoshi Hirabayashi and Michitaka Kohno \\ Dept. of Orthop. Surg., School of Med., Keio-Gijuku University
}

67 cases of discogram on the cervical spondylosis out of 95 cases of cervical disc lesion are discussed.

The degeneration, posterior protrusion and lateral protrusion of the cervical discs are divided into 4 groups from normal to severe according 
to their discogram.

Cervical discogram reflects the change of discs in the great extent and precedes the sign of bony changes on X-ray findings.

Physiological changes are elicited at the later age of 20 th showing the degenerative changes invading into the unco-vertebral joint.

Neighbouring discs up and down the involved disc also revealed the degenerative changes to some extent.

Dynamic change of the disc in extension and flexion position suggests the relationship between the development of clinical feature of the cervical disc lesion and cervical mobility.

\title{
117. Mechanism of Neurological Manifestations of Cervical Disc Lesions
}

\author{
M. Tsuru, T. Takeda, K. Yada, and H. Takamura \\ Department of Neurosurgery, Hokkaido Univ. School of Med.
}

During the past six years, we have treated 180 cases of cervical disc lesions at our department. This presentation is based on an analysis of 44 cases of which lesions were surgically verified.

Although both spondylosis and disc herniation (soft disc) have been considered to have same basic pathology, namely degeneration and protrusion of the disc material, their neurological manifestations are quite different. In cases of cervical spondylosis, the commonest clinical findings are atrophy and motor weakness of the upper extremities which is most marked in the intrinsic muscles of the hands, hyperactive reflexes of all four extremities including biceps and triceps reflexes, both radicular and cord types of sensory disturbanses, and spastic paresis of the lower extremities. Most of these signs were also noted in the cases of soft disc hernia, however, in the latter cases cord signs were tended to be Brown-Sequard type and the deep tendon reflexes of the upper extremities were not increased but decreased at the corresponding level of the lesion.

Operative results which were analyzed based on long term follow-up study obtained 77 per cent of total operative cases also gave us very interesting informations as regard to the mechanism of neurological manifestations of this type of the lesions. Removal of protluded disc material in cases of soft disc hernia resulted in complete recovery or marked improvements 